\title{
Friedensstifterinnen und Schutz vor sexualisierter Kriegs- gewalt in Afrika - internationale politische Kontexte und lokale Probleme
}

\author{
Rita Schäfer*
}

\begin{abstract}
Women's organisations in Africa demand peace and try to participate in peace negotiations. Their main aim is the reduction of sexualized violence. They draw on the UN Resolution 1325 "Women, Peace and Security" for their political lobbying. On the one hand this resolution promotes women's participation in peace and democratization processes. On the other hand it conceptualizes women mainly as victims of violence. Women's organisations in post-conflict societies are confronted with this dilemma. In addition, they have to cope with serious problems to enforce the resolution, as the case studies of the Democratic Republic of Congo and Liberia reveal.
\end{abstract}

Keywords: UN Resolution 1325, peace processes, women's organisations in Africa, post-conflict societies, security sector reform VN-Resolution 1325, Friedensprozesse, afrikanische Frauenorganisationen, Nachkriegsgesellschaften, Sicherheitssektorreform

\section{Zeithistorische politische Kontexte}

In der Debatte über nachhaltige Friedensprozesse in afrikanischen Post-Conflict-Gesellschaften hat die Auseinandersetzung mit den Friedenspotenzialen zivilgesellschaftlicher Gruppierungen in den letzten Jahren an Bedeutung gewonnen. Hohen Stellenwert hat die Ausrichtung auf die von Frauenorganisationen geleistete konkrete Friedensarbeit. Impulsgebend war die UN-Resolution 1325 zu Frauen, Frieden und Sicherheit, die am 31.Oktober 2000 einstimmig vom UN-Sicherheitsrat verabschiedet wurde. Sie kombiniert den Schutz von Frauen vor Gewalt mit dem Anspruch der Strafverfolgung und Prävention sexualisierter Kriegsgewalt sowie einer stärkeren Beteiligung von Frauen an Friedensprozessen. Die Inhalte und die Entstehung dieser Resolution gingen keineswegs nur auf UN-Vertreter zurück; vielmehr wirkten daran auch afrikanische Frauenorganisationen und internationale Frauenrechtsaktivistinnen mit.

Dieser Beitrag setzt sich mit der normativen Ebene der UN-Sicherheitsratsresolution 1325 auseinander und ordnet ihre Entstehung in die Geschichte der Frauenfriedenspolitik in Afrika ein. Darüber hinaus zeigt er die Kluft auf zwischen zentralen Inhalten der Resolution sowie den daraus abgeleiteten nationalen Aktionsplänen und deren mangelhafter Umsetzung auf lokaler Ebene. Die in der Resolution nicht aufgelösten Gegensätze, d.h. die reaktive Aufarbeitung sexualisierter Gewalt einerseits und die Gewaltprävention sowie die konstruktive Friedensarbeit andererseits, stellt die von Frauen gegründeten Friedensorganisationen vor große Herausforderungen. Hinzu kommt das Problem, dass Gewaltprävention und Friedensarbeit viele Politikbereiche umfassen, die weit über geschlechtsspezifische Gewalterfahrungen hinausgehen. Zudem müssen die Friedensaktivistinnen auf unterschiedlichen Ebenen aktiv werden und Einfluss ausüben: Zum einen müssen sie konkrete Lobbyarbeit bei Regierungen betreiben, um die Verankerung von Frauenrechten und internationalen Rechtsnormen in

Rita Schäfer ist Ethnologin und freiberufliche Wissenschaftlerin. neuen nationalen Gesetzgebungen durchzusetzen oder die Implementierung landesweiter Aktionspläne voranzubringen. Zum anderen müssen sie auf lokaler Ebene die Menschen für geschlechtsspezifische Gewaltprobleme als Kriegsfolgen sensibilisieren. Denn hier stehen vielfach selektive Interpretationen von Kultur und Tradition durch alte und neue Eliten veränderten Normen und Orientierungen entgegen, erstgenannte verstärken gewaltgeprägte Geschlechterhierarchien in den Nachkriegsgesellschaften.

Die Grundlagen für den Text zur UN-Sicherheitsratsresolution 1325 wurden im Mai 2000 von Friedensaktivistinnen auf dem sogenannten „Windhoek-Seminar“ erarbeitet. Das Seminar wurde unter dem Titel „Mainstreaming a Gender Perspective in Multidimensional Peace Support Operations“ von der UNHauptabteilung Friedenssicherungseinsätze (UN DPKO) organisiert und durchgeführt. Das Treffen mündete in die „Windhoek-Erklärung“ und den „namibischen Aktionsplan“ (Barnes 2011: 18; Olsson/Tryggestad 2001). Aufgrund der Geschichte Namibias war die Hauptstadt Windhoek speziell als Seminarort ausgewählt worden. Das Land war erst 1990 nach einem jahrelangen und blutigen Bürgerkrieg von Südafrika in die Unabhängigkeit entlassen worden. An diesem Guerillakrieg waren auch zahlreiche Mädchen und junge Frauen als Kombattantinnen beteiligt, die von der South West African People's Organisation (SWAPO) gezielt mit emanzipatorischen Parolen angeworben worden waren (Cleaver/Wallace 1990:81ff.). Doch ähnlich wie in anderen bewaffneten Befreiungsbewegungen im südlichen Afrika widersprach die Kriegsrealität der Kämpferinnen diesen Ideen. Sexualisierte Gewalt durch Kommandanten war weit verbreitet. Zudem wurden Frauen nach dem Ende des Krieges von Entwaffnungs-, Demobilisierungs- und Reintegrationsprogrammen, die von der Unterstützungseinheit der Vereinten Nationen für die Übergangszeit (UNTAG) begleitet wurden, ausgeschlossen. Die emanzipatorischen Absichtserklärungen der SWAPO, die 1990 schließlich Regierungspartei wurde, fanden keine Umsetzung in der Politik. Auch die Ernennung einer Frauenministerin und der Einzug einiger weiblicher Abgeordneter ins namibische Parlament änderten daran nichts. 
Bis heute müssen unabhängige Frauenrechtsorganisationen, in denen u.a. ehemalige Kämpferinnen mitarbeiten, frauenspezifische Rechtsnormen und deren Umsetzung beharrlich einfordern. Noch immer ist der Anteil an ehelicher und sexualisierter Gewalt in der namibischen Gesellschaft sehr hoch, wobei Vergewaltigungen zudem zu einer rasanten Ausbreitung von HIV beitragen. Die Täter werden mehrheitlich nicht strafrechtlich verfolgt, weil ihre gewalttätigen Übergriffe in der Gesellschaft oft nicht als Straftaten angesehen werden. Polizisten und Richter teilen diese weit verbreiteten sexistischen Frauenbilder und Vorstellungen; sie sind zumeist ungebrochen aus den Kriegsjahren in die Nachkriegszeit übernommen worden (Schäfer 2008: 58ff.). Das UN-DPKO Seminar, an dem auch einige ExKämpferinnen - nunmehr eingebunden in Frauenrechts- oder Friedensorganisationen - teilnahmen, im Mai 2000 in Windhoek abzuhalten, war auch als Unterstützung für die Durchsetzung von Frauenrechten im Land gedacht.

Dieses Seminar steht in engem zeitlichen Zusammenhang mit der UN-Sicherheitsratsresolution 1325. Vorausgegangen war eine Debatte im UN-Sicherheitsrat anlässlich des internationalen Frauentags am 8. März 2000. Hier wurden die Situation von Frauen in Kriegen sowie ein neues Sicherheitskonzept erörtert. Schon zwei Monate später fand das Seminar in Namibia statt. Im Oktober 2000 kam es dann zur Verabschiedung der UN-Sicherheitsratsresolution 1325. Anlässlich des Weltfrauentags 2001 bekräftigte die UN-Generalversammlung die Bedeutung dieser Resolution und forderte unabhängige Frauenorganisationen ausdrücklich auf, ihre Umsetzung kritisch zu begleiten.

Afrikanische Friedens- und Frauenrechtsaktivistinnen hatten schon frühere UN-Veranstaltungen genutzt, um ihre Interessen zu artikulieren. Hierzu zählt die internationale Abschlusskonferenz der Weltfrauendekade in der kenianischen Hauptstadt Nairobi im Jahr 1985. Die dort vertretenen Frauenorganisationen verlangten ein Ende der Kriege auf ihrem Kontinent und nahmen dabei ausdrücklich auf das von den UN gewählte Motto „Women, Development and Peace“ Bezug (Tripp 2009: 3ff.).

\section{Friedensaktivistinnen in Afrika}

Nach dem Ende des Ost-West-Konflikts wurden etliche Demokratisierungsbewegungen in Afrika gewaltsam niedergeschlagen; Frauen- und Mädchen wurden zu Zielscheiben der Kriegsherren und deren Kämpfer bzw. Soldaten. Die massenhafte sexualisierte Gewalt mobilisierte im Gegenzug Friedensaktivistinnen, die neue Netzwerke bildeten. Hierzu zählte die 1994 in Dakar gegründete Federation of African Women Peace Networks, die gemeinsame Forderungen für die Weltfrauenkonferenz in Peking 1995 erarbeitete (Tripp et al. 2009: 68). Die Aktionsplattform von Peking enthielt einen umfangreichen Forderungskatalog zum Schutz von Frauen in Kriegen und zur Beteiligung von Frauen an Friedensprozessen. ${ }^{1}$ Das Abschlussdokument von Peking stellte einen wichtigen Referenzpunkt für weitere Schritte in diese Richtung dar. Es wurde von vielen afrikanischen Regierungen unterzeichnet und auch die

1 http://www.peacebuildingportal.org/index.asp?pgid=9\&org=2854
Friedenskonferenz afrikanischer Frauenorganisationen 1999 in Sansibar bezog sich darauf (Karame 2004). ${ }^{2}$ So wurde es zu einer wichtigen Grundlage für das Windhoek-Seminar und den Text der Resolution 1325.

Die Mitwirkung von Frauen in Friedensprozessen und ein Ende der sexualisierten Kriegsgewalt zählen zu den Zielen der Afrikanischen Dekade für Frauen 2010-2020, die von der Afrikanischen Union (AU) ausgerufen wurde. Sie nimmt auf die Mitwirkung afrikanischer Frauenorganisationen an der Weltfrauendekade (1975-1985), die Weltfrauenkonferenzen und die UN-Resolution 1325 Bezug und wurde im Rahmen des Treffens der UN-Kommission für die Rechtsstellung der Frau zum Weltfrauentag 2010 in New York vorgestellt. ${ }^{3}$ Problematisch ist jedoch, dass all diese Abkommen völkerrechtlich nicht bindend sind und es den jeweiligen Regierungen überlassen ist, sie umzusetzen. Umso wichtiger ist die oft mühsame rechtspolitische Lobbyarbeit von Frauenrechts- und Friedensorganisationen. Gleichzeitig ist ihre kritische Auseinandersetzung mit der Regierungspolitik in etlichen Ländern mit schwachen Oppositionsparteien bedeutend (Tripp et al. 2009: 79f.).

\section{Die UN-Resolution 1325 und Folge- resolutionen}

Die UN-Resolution 1325 ist ein wichtiges Lobbyinstrument für Frauenrechts- und Friedensorganisationen. ${ }^{4}$ In der friedenspolitischen Debatte gilt sie oft als Meilenstein; hingegen äußern kritische Friedenswissenschaftlerinnen Zweifel an dieser Einschätzung. Sie bemängeln, dass weder die UN-Resolution 1325 noch die darauf aufbauenden Folgeresolutionen 1820, 1888, 1889, 1960 aus den Jahren 2008 bis 2010 wirkliche Verbesserungen gebracht haben, obwohl diese Teilaspekte der UN-Resolution 1325 mit speziellen Forderungskatalogen erweiterten. Dieser Beitrag konzentriert sich auf die Forderungen, die sexualisierte (Nach)Kriegsgewalt und friedenspolitisch aktive Frauenorganisationen betreffen. So verlangen die UN-Resolutionen 1820 und 1888 die energische Bekämpfung sexualisierter Gewalt und die Resolution 1889 fordert eine stärkere Mitwirkung von Frauen an Friedensprozessen. Zudem soll der UN-Generalsekretär einen Indikatorenkatalog zur Beurteilung für die Umsetzung der Resolution vorlegen (Barnes 2011: 27ff.). Die UN-Resolution 1960 unterstreicht die Bedeutung des Internationalen Strafgerichtshofs zur strafrechtlichen Verfolgung von Vergewaltigungen. Schließlich hatte das Rom-Statut 1998 sexualisierte Kriegsgewalt als Verbrechen gegen die Menschlichkeit und als Kriegsverbrechen in den Straftatbestand aufgenommen (Sjoberg/Via 2010).

Das Grunddilemma der Resolutionen besteht darin, dass sie aufbauend auf den Text der UN-Resolution 1325 - einerseits Täter bestrafen und Frauen vor Gewalt schützen wollen, also einem Opferdiskurs folgen und diesen betonen. Andererseits zielen sie darauf ab, die politische Partizipation von Frauen zu

\footnotetext{
2 http://www.unesco.org/cpp/uk/projects/women_organize_for_peace_in_africa.htm

3 http://www.pambazuka.org/aumonitor/AUMONITOR/comments/african womens_decade/

4 http://www.un.org/events/res_1325e.pdf
} 
verbessern. Hierdurch sollen nachhaltige Friedensprozesse gestärkt werden. Diesen Gegensatz von paternalistischen Schutzpflichten, insbesondere durch den Staat, und dem eigenständigen politischen Handeln von Frauen löst die UN nicht auf (Olonisakin/Okech 2011). Kritische Friedenswissenschaftlerinnen werfen dem UN-Sicherheitsrat vor, den Opferdiskurs zu bedienen (Puechguirbal 2010:172ff.; Carpenter 2006). Aber auch manche Frauenorganisationen tragen zur Intensivierung des Opferdiskurses bei, indem sie in ihrer Lobbyarbeit Frauen und Mädchen vor allem als Opfer bzw. zu Beschützende präsentieren und ihre Regierungen bzw. staatliche Institutionen damit in die Pflicht nehmen wollen (Chandler/Wang/Fuller 2010). Wie problematisch dieses Vorgehen ist, wird deutlich, wenn Regierungen ihre Schutzpflichten selektiv auslegen und beispielsweise mit partikularen Machtinteressen verbinden (vgl. Jacobs/Jacobson/Marchbank 2000). Ein kritischer Blick auf die Resolutionen zeigt, dass staatliche Institutionen in etlichen Nachkriegsgesellschaften Geschlechterhierarchien verstärken, zumal deren Mitarbeiter oft martialische Maskulinitäts- sowie lokale Geschlechterkonstrukte verinnerlicht haben und ihr Handeln daran orientieren, obwohl diese Vorstellungen den neuen internationalen normativen Vorgaben und den nationalen Rechts- und Sicherheitssektorreformen entgegenstehen (Wisotzki 2011:21f.).

Auch die Idealisierung von Frauen als Friedensstifterinnen könne für politische Propaganda ausgenutzt werden, warnen zahlreiche Friedensforscherinnen. Zudem bestehe die Gefahr, dass Frauenorganisationen sich selbst einen Fallstrick legen, wenn sie einerseits die Friedensleistungen von Frauen hervorheben und andererseits die Gewaltbedrohungen durch Männer betonen. Dies führe zu polarisierten Geschlechterstereotypen (Wibben 2011: 21ff.; Kaufman/Williams 2010).

Darüber hinaus kritisieren Friedenswissenschaftlerinnen die Resolutionstexte, weil diese Gender mit Frauen gleichsetzen und Gender-Mainstreaming in Friedensprozessen auf Frauenquoten reduzieren (Shepherd 2010: 160ff.). Die UN-Resolutionen zu Frauen, Frieden und Sicherheit haben einen female bias (Barnes 2011: 20ff.). Ignoriert werden kriegsgeprägte Männlichkeitsvorstellungen, die Militarisierung von Gesellschaften und deren Folgewirkungen für die Verbreitung und Akzeptanz von geschlechtsspezifischen oder anderen Gewaltformen. Auch die Tatsache, dass Männer und Jungen sowohl Täter als auch Opfer sein können, findet keine Berücksichtigung (Myrttinen 2003: 37ff.). Problematisch daran ist, dass Männer, die ihre vielfältigen und widersprüchlichen Gewalterfahrungen in Kriegen nicht aufarbeiten können, Gewaltmuster in die Nachkriegsgesellschaften unreflektiert übernehmen und oft zu geschlechtsspezifischen Gewaltformen greifen, um ihre Männlichkeit zu bestätigen (Eifler/Seifert 2009). Dies betrifft keineswegs nur demobilisierte und perspektivlose Ex-Kämpfer, sondern auch Zivilisten, die Gewalt erfahren haben.

Außerdem werden die verschiedenen Kriegserfahrungen von Frauen und Mädchen unterschiedlichen Alters oder Status nicht differenziert. Diese können neben sexualisierter und körperlicher Gewalt, Zerstörung von Ernten und Häusern, Flucht und Vertreibung umfassen. Frauen als (Mit)täterinnen tauchen in den Resolutionen nur verdeckt auf, nämlich als Kindersoldatinnen. Damit werden die oft widersprüchlichen Rollen von
Mädchen und Frauen in Kriegen ignoriert, die sowohl Kämpferinnen als auch Gewaltopfer sind und dadurch gegen tradierte weibliche Rollenmodelle verstoßen (Moser/Clark 2001).

\section{Liberia und D.R. Kongo}

Grundlegende Probleme bei der Umsetzung der UN-Resolutionen zu Frauen, Frieden und Sicherheit lassen sich an den Beispielen Liberia und der Demokratischen Republik Kongo exemplarisch illustrieren. Beide Länder haben nationale Aktionspläne zur UN-Resolution 1325 erlassen. Dennoch werden sie wegen ihrer unterschiedlichen Umsetzung in der gegenwärtigen frauen- und friedenspolitischen Debatte als Gegenpole kategorisiert. Liberia gilt wegen seiner Gesetzesnovellen, Rechtsreformen und Frauenförderprogramme, die von der ersten Präsidentin Afrikas Ellen Johnson-Sirleaf vorangebracht wurden, als Musterland für geschlechtergerechte Nachkriegsentwicklungen. Demgegenüber haben Gender-Themen in der Demokratischen Republik Kongo unter Präsident Josef Kabila keine politische Bedeutung; die Gender-Ministerin und die Parlamentarierinnen sind weitgehend marginalisiert.

Trotz der unterschiedlichen frauenpolitischen Bilanzen haben beide Länder viele Gemeinsamkeiten. Dazu zählen die schleppenden Sicherheitssektorreformen und hohe Raten an sexualisierter Gewalt, insbesondere in ländlichen Gebieten. Dort versagen staatliche Institutionen; so legitimieren z.B. Juristen und traditionelle Autoritäten, die Recht sprechen dürfen, gewaltgeprägte Geschlechterhierarchien, indem sie Urteile nach Kultur und Tradition fällen, die oftmals internationalen normativen Vorgaben und nationalen Rechtsreformen widersprechen. Lokale Frauenrechtsorganisationen fordern grundlegende Reformen von Polizei und Justiz, um der Straflosigkeit bei sexualisierten Gewaltverbrechen Einhalt zu gebieten. Liberianische Aktivistinnen müssen sich darüber hinaus mit (neo-)traditionalistischen Amtsträgerinnen in ländlichen Gebieten auseinandersetzen, deren Vorstellungen vom gesellschaftlichen Wiederaufbau auf Mädchenbeschneidungen und der Unterordnung von Frauen basieren.

\section{Kriegs- und Nachkriegsprobleme in Liberia}

In Liberia herrschte zwischen 1989 und 2003 ein äußerst brutaler Bürgerkrieg, bei dem Regierungstruppen gegen verschiedene Guerillaorganisationen kämpften, die sich auch gegenseitig bekriegten. Zu den Kriegsursachen zählten große wirtschaftliche und soziale Ungleichheiten sowie die Marginalisierung und Verarmung ganzer Bevölkerungsgruppen. Liberia wurde bereits 1847 eine eigenständige Republik, in der freigekaufte Sklaven aus Amerika angesiedelt wurden. Diese Americo-Liberianer bildeten eine neue Elite in der neugegründeten Hafen- und Hauptstadt Monrovia; sie beuteten die natürlichen Ressourcen im Landesinneren auf Kosten der dortigen Bevölkerung aus. Ab 1980 baute Diktator Samuel Do eine brutale Militärherrschaft auf; zwar postulierte er, die Interessen lokaler Ethnien aus dem Landesinneren zu vertreten, vernachlässigte aber die gesamte ländliche Infrastruktur einschließlich 
des Bildungs- und Gesundheitswesens. Seine Ermordung 1989 beendete keineswegs die Willkürherrschaft, vielmehr war sie der Auftakt zu einem vierzehnjährigen Guerillakrieg (Schäfer 2008: 181ff.). Zwangsrekrutierte Mädchen und junge Frauen stellten etwa ein Drittel der Guerillaeinheiten; sie mussten unterschiedliche Aufgaben übernehmen und zahllose wurden vergewaltigt.

Um den Kriegsgräueln ein Ende zu bereiten, gründeten Stadtbewohnerinnen in Monrovia die Liberian Women's Initiative (LWI). Daran wirkten maßgeblich christliche und moslemische Frauen mit - viele waren Lehrerinnen und Ärztinnen. Im gleichen Jahr schlossen sich liberianische Juristinnen zur Association of Female Lawyers of Liberia (AFLL) zusammen; sie verlangten das Ende des Krieges und die Einhaltung von Frauenrechten. Sie verschafften ihren Forderungen beim afrikanischen Vorbereitungstreffen zur Weltfrauenkonferenz in Peking in Dakar 1994 Gehör. Angesichts der grenzüberschreitenden Ausmaße des Krieges gründeten ranghohe und politisch einflussreiche Frauen aus Liberia, Sierra Leone und Guinea im Jahr 2000 das Mano River Women's Peace Network (MARWOPNET). Ende 2001 kam das Women in Peacebuilding Network (WIPNET) hinzu, ein Zweig des West Africa Network for Peacebuilding (WANEP). Es wollte Frauen unterschiedlichen Alters und Status einen und mobilisierte vor allem junge und arme Frauen, die aus ländlichen Kampfgebieten nach Monrovia geflohen waren.

WIPNET organisierte Massendemonstrationen in der Hauptstadt, an denen viele Mütter teilnahmen, die sich für Frieden einsetzten. ${ }^{5}$ Ein Friedensabkommen, das auch tatsächlich eingehalten wurde, kam erst am 18. August 2003 in der ghanaischen Hauptstadt Accra zustande. Mitte 2003 waren WIPNET-Vertreterinnen zu den Friedensverhandlungen nach Accra gereist. Ihnen wurde der Zutritt zu den offiziellen Verhandlungen verweigert, deshalb versuchten sie vor und nach den Sitzungen, persönlichen Druck auf die einzelnen Verhandlungsteilnehmer auszuüben. Sie drohten den einzelnen Warlords, sich öffentlich zu entkleiden, falls sie nicht einlenkten. Angesichts des internationalen medialen Interesses an den Friedensverhandlungen hätte dies einen nachhaltigen Gesichtsverlust für jeden einzelnen Milizenchef bedeutet. Nur ausgewählte MARWOPNET-Vertreterinnen, die zur politischen Elite zählten, waren schon im Vorfeld zu den Friedensverhandlungen eingeladen worden. Sie bemühten sich, die Probleme der Ex-Kombattantinnen als Kriegsopfer im Friedensabkommen zu berücksichtigen, was aber wegen konzeptioneller, institutioneller und personeller Mängel nur ansatzweise gelang. Die Entwaffnungs-, Demobilisierungs- und Regintegrationsprogramme, denen Vorstellungen von martialischer Männlichkeit zugrunde lagen, orientierten sich vor allem an jungen Kämpfern. Ex-Kombattantinnen hatten kaum Partizipationsmöglichkeiten, ihre widersprüchlichen Kriegserfahrungen als (Mit)Täterinnen und Gewaltopfer blieben unbeachtet. Friedens- und Frauenrechtsaktivistinnen hatten immer größere

5 Die WIPNET-Mitbegründerin und Koordinatorin der Massenproteste Leymah Gbowee wird für ihre couragierte Arbeit mit dem Friedensnobelpreis 2011 ausgezeichnet. Die zwei anderen Preisträgerinnen sind die liberianische Präsidentin Ellen Johnson-Sirleaf und die jemenitische Bürgerrechtlerin Tawakkul Karman.
Probleme, gemeinsame Positionen zu formulieren. Bereits während des Krieges mussten sie gravierende Differenzen zwischen Frauen unterschiedlicher Herkunft und Bildung überbrücken. Nachdem das verbindende Ziel, den Krieg zu beenden, erreicht worden war, verstrickten sie sich in Interessenkonflikten und schmälerten so ihren politischen Einfluss.

Unterstützung durch die Vereinten Nationen, z.B. durch UNIFEM-Gelder für einzelne Frauenorganisationen oder den Einsatz einer Gender-Beraterin in der United Nations Mission in Liberia (UNMIL), konnten das nicht verhindern. Die GenderBeraterin kooperierte zwar punktuell und temporär mit Vertreterinnen der Juristinnenvereinigung AFLL (Wamai 2011: 54ff.). Sie war jedoch auf sich allein gestellt und institutionell marginalisiert, daher konnte sie weder die Mängel der Demobilisierungsprogramme beheben noch die strukturellen GenderProbleme der UN-Mission lösen. Diese Probleme zeigten sich u.a. darin, dass UNMIL-Soldaten immer wieder junge Liberianerinnen missbrauchten.

Nach dem Sieg bei den Präsidentschaftswahlen und ihrer Amtseinführung 2006 setzte sich die Präsidentin Ellen Johnson-Sirleaf dafür ein, dass eine Reihe von Gesetzesnovellen verabschiedet wurde und in Kraft trat. Auch den nationalen Aktionsplan zur Umsetzung der UN-Resolution 1325 brachte sie maßgeblich voran; für seine Umsetzung ist das Gender-Ministerium zuständig. Dieser Plan fordert Nichtregierungsorganisationen auf, die Implementierung kritisch zu begleiten. Sie bilden wegen ihrer internen Konflikte zwar keine gemeinsame politische Lobby, dennoch fordern einige beharrlich die Umsetzung der Sicherheitssektorreform und beziehen sich dabei auf den nationalen Aktionsplan zur UN-Resolution 1325. Besonders schwierig ist die Arbeit von Frauenorganisationen, die in den infrastrukturell schlecht erschlossenen und wirtschaftlich desolaten früheren Kampfgebieten tätig sind, wo sexualisierte Gewalt als Nachkriegsproblem virulent ist. Die wenigen dort eingesetzten Polizisten oder Richter sind schlecht ausgebildet und ausgestattet, überfordert und korrupt. Deshalb können sich Vergewaltiger freikaufen (Nagelhuis/de Cavallo 2009: 26ff.).

Lokale Frauenrechtsorganisationen wie Medica Mondiale Liberia, die sich auf die nationalen Reformvorgaben sowie die internationalen Resolutionen zu Frauen, Frieden und Sicherheit berufen, beklagen diese Missstände. Gleichzeitig sind sie mit einem selektiven Traditionalismus konfrontiert, denn mancherorts urteilen Familiengerichte über Vergewaltigungen und zwingen die Opfer zur Ehe mit den Peinigern. Auch den Rekurs auf den Traditionalismus im Wahlkampf 2005 prangerten die Aktivistinnen an. Das betraf Reisgeschenke, die Politikergattinnen vor den Wahlen an lokale Beschneiderinnen verteilten. Diese sollten Wählerinnen mobilisieren, denn sie besetzten nach wie vor Machtpositionen in lokalen Frauenbünden (Fuest 2008: 205ff.). Offiziell sind genitale Operationen nur an minderjährigen Mädchen verboten. Solche Praktiken stehen aber im Widerspruch zum Gewaltschutz, den Gesetzesnovellen zu Frauenrechten und dem Nationalen Aktionsplan zur UN-Resolution 1325. Von der Gender-Ministerin haben die Aktivistinnen keine Unterstützung zu erwarten, zumal sie erst Mitte 2011 die Bewahrung von Traditionen verteidigte. Es bleibt zu hoffen, dass Ellen Johnson-Sirleaf, die am 10. Dezember 2011 gemeinsam mit der liberianischen Friedensaktivistin Leymah Gbowee 
und der jemenitischen Bürgerrechtlerin Tawakkul Karman den Friedensnobelpreis 2011 erhalten wird, nach ihrer Wiederwahl im November 2011 weiterhin die Leitlinien zur Frauenförderpolitik vorgeben wird.

\section{Kriegs- und Nachkriegskontexte in der D.R. Kongo}

In der D.R. Kongo werden am 28. November 2011 Parlamentsund Präsidentschaftswahlen abgehalten. 2006 hatte erste Urnengang nach den Kriegen stattgefunden, die zwischen 1998 und 2003 ganz Zentralafrika erschütterten. Während der Kriegsjahre hatte die kongolesische Armee - durch Militärhilfe aus Simbabwe, Namibia und Angola - verschiedene konkurrierende Guerillagruppen bekämpft. Diese rangen wiederum untereinander gewaltsam um Macht und Ressourcenkontrolle. Sie bestanden mehrheitlich aus zwangsrekrutierten jungen Kämpfern. Mädchen bildeten nur eine Minderheit und wurden vor allem als Sexsklavinnen missbraucht. Bei den schleppend durchgeführten Demobilisierungsprogrammen nach dem Kriegsende wurden sie weitgehend ignoriert. Die von unterschiedlichen Milizen systematisch als Kriegstaktik gegen Zivilistinnen eingesetzte sexualisierte Gewalt wurde im Nachkriegskontext kaum strafrechtlich aufgearbeitet (Amnesty International 2004: 7ff.). Das konnte die neue Gender-Ministerin, deren politischer Einfluss sehr begrenzt ist, nicht ändern.

Kongolesische Frauenrechtsaktivistinnen, die wie die Mitarbeiterinnen der Organisation Promotion et Auppui aux Initiatives Féminines (PAIF) ihre Arbeit als Beitrag zu tragfähigen Friedensprozessen betrachten, haben große Sorge, dass es während und nach den Wahlen wieder zu gewalttätigen Ausschreitungen kommen wird, bei denen Frauen und Mädchen vergewaltigt werden. Das Wahlrecht ist nicht verfassungskonform und Wählerlisten wurden nicht aktualisiert, sodass vor allem junge Wähler und Wählerinnen nicht registriert sind. Die Zahl der Wahllokale wurde drastisch reduziert, was dazu führt, dass insbesondere zahllose Landbewohnerinnen ihr Wahlrecht nicht ausüben können. Die verkehrstechnische Infrastruktur ist in einem katastrophalen Zustand und schon jetzt ist es wegen gewaltsamer Übergriffe für Frauen gefährlich, in ländlichen Gebieten Fußwege zu nutzen. Frauenrechtsorganisationen wie PAIF haben vorgeschlagen, mobile Wahlstationen dorthin zu bringen. Eigentlich verpflichten die Verfassung und der Nationale Aktionsplan zur UN-Resolution 1325 die kongolesische Regierung dazu, die politische Partizipation von Frauen zu fördern und sie vor Gewalt zu schützen. Jedoch ist die Lebensrealität der Bevölkerung in den Kivu-Provinzen weit von Demokratisierung und Rechtsstaatlichkeit entfernt; hier haben marodierende und sich bekämpfende Milizen das Sagen.

PAIF prangert seit Jahren die Ausbeutung und Gewalt beim Coltan-Abbau und -Handel an. Neben Raub von Vieh, Zerstörung der Ernten, Plünderungen, Erpressungen, Zwangsarbeit und körperlicher Gewalt gelten Vergewaltigungen als effektives Mittel zur Gebietseroberung und Vertreibung. Wegen der katastrophalen Gesundheitsversorgung und des mangelnden Medikamentenzugangs steigt die Mutter-Kind-Übertragung des HI-Virus; AIDS hat eine hohe Säuglings-, Kinder- und Müt- tersterblichkeit zur Folge. Immer wieder suchen Landbewohnerinnen und Landbewohner in Flüchtlingslagern Schutz, wo sie von Nahrungsmittelhilfe abhängig werden. Die dortige Perspektivlosigkeit fördert häusliche Gewalt und sexuellen Missbrauch. Das kongolesische Familienrecht und selektive Interpretationen vorkolonialer Rechtsvorstellungen durch (neo-) traditionelle und staatliche Autoritäten gewähren Männern umfassende Machtbefugnisse über ihre Frauen und Töchter. Alle anderen Machtbereiche haben sie durch die Milizen verloren (Dolan 2010). Sogar die Arbeit als illegale Minenarbeiter bringt den Männern wenig Lohn; an den lukrativen Mineralien bereichern sich Zwischenhändler und Hintermänner. Die Straffreiheit für die Coltan- und Waffenschmuggler und die Straflosigkeit bei Vergewaltigungen belegen, dass der Rechtsstaat in den Kivu-Provinzen kaum präsent ist.

Mit diesen Problemen sind nicht nur die PAIF-Aktivistinnen, sondern auch andere kongolesische Frauenorganisationen konfrontiert. Im Vorfeld der Friedensverhandlungen 2002 kamen sie zusammen, um gemeinsame friedenspolitische Ziele zu formulieren. Allerdings erschwerten Interessenunterschiede und die mangelnde Infrastruktur eine kontinuierliche Kooperation. So handelt es sich mehrheitlich um punktuelle Initiativen, an deren lockerer Vernetzung sich PAIF beteiligt. Die Synergy des Femmes ist ein Zusammenschluss von 35 Organisationen, die in Nord-Kivu Gewaltopfern psycho-soziale und rechtliche Hilfe bietet. In Süd-Kivu vermitteln die Réseau des Femmes pour la Défense des Droits et la Paix und die Réseau des Femmes pour un Développement Associatif Rechtsinformationen; lokale Radiostationen sorgen für die Verbreitung in abgelegenen Gebieten. Gemeinsame Lobby- und Aufklärungsarbeit können überlebenswichtig sein, denn die Frauenrechtsaktivistinnen werden immer wieder bedroht und eingeschüchtert, weil sie Gewaltstrukturen ans Tageslicht bringen (Schäfer 2008: 372ff.). Weder Polizei noch Justiz schützen sie. Drastischer Personalmangel, schlechte Ausbildung und Entlohnung, Korruption und Ineffizienz prägen diese Institutionen. Namentlich bekannte Gewalttäter kaufen sich frei, falls sie überhaupt vor Gericht gestellt werden. Auch das kongolesische Militär ist schlecht ausgestattet und korrupt, oft kommen Soldzahlungen nicht bei den Soldaten an. Disziplin und Truppenmoral sind durch die übereilte Integration ehemaliger Milizionäre in die staatliche Armee drastisch gesunken. Unklare Befehlsstrukturen und die Ignoranz der höchsten Vorgesetzten im fernen Kinshasa über die chaotischen Zustände verärgern kongolesische Soldaten, etliche vergewaltigen aus Frustration (Baaz/Stern 2009: 495ff.). Mehrheitlich haben sie nicht mit Strafverfolgung zu rechnen.

Die Unfähigkeit staatlicher Institutionen ist nicht allein auf die Kriege Ende der 1990er Jahre zurückzuführen. Sie geht auch auf die über 30-jährige Mobutu-Diktatur und die belgische Kolonialherrschaft seit dem 19. Jahrhundert zurück, zumal die Belgier und Mobutu Gewalt als Mittel der Herrschaftssicherung nutzten. Viele Kongolesinnen und Kongolesen kannten die zumeist männlichen Vertreter staatlicher Institutionen nur als Repräsentanten eines Repressionsapparats. Umso wichtiger ist die Arbeit zivilgesellschaftlicher Organisationen wie PAIF, die Rechtsinformationen bieten und Strukturreformen der staatlichen Institutionen verlangen, wobei sie sich auf nationale Rechtsgrundlagen und internationale Abkommen beziehen, zu 
deren Einhaltung die kongolesische Regierung sich verpflichtet hat. Allerdings ist die Gender-Ministerin keine durchsetzungsfähige Allianzpartnerin und auch die UN-Friedenstruppe hat sich wiederholt als unfähig erwiesen, Frauen zu schützen. Vielmehr ist sexueller Missbrauch durch Blauhelmsoldaten seit Jahren ein Problem.

\section{Fazit}

Die Fallbeispiele illustrieren, wie schwierig es ist, die nationalen Aktionspläne zur UN-Sicherheitsratsresolution 1325 zu Frauen, Frieden und Sicherheit umzusetzen und die normativen Vorgaben ihrer Folgeresolutionen zu erfüllen. Besonders virulent ist dies in den von gewaltsamen Ressourcenkonflikten geprägten Kivu-Provinzen, wo die mangelnde Präsenz staatlicher Institutionen sowie die Inkompetenz und der Sexismus ihrer Mitarbeiter und lokaler Autoritäten Strukturprobleme sind, mit deren Lösung die schwache Gender-Ministerin überfordert ist. Selbst in Liberia, das offiziell eine staatliche Gender-Politik verfolgt, verhindern die schleppenden und unzureichenden Reformen von Polizei und Justiz sowie der Machtgewinn alter lokaler Eliten - auch ranghoher weiblicher Autoritäten - , dass Frauen in ländlichen Gebieten vor Gewaltübergriffen geschützt werden. Vielmehr verbreiten diese Autoritäten selektive Reinterpretationen von Traditionen zur Rechtfertigung von Gewalt und Unrecht. Die politische Partizipation von Frauen wird zum Spielball des Machterhalts lokaler Eliten, was sogar die GenderMinisterin mitträgt.

In beiden Fällen prangern Frauenrechtsorganisationen die Missstände an. Sie berufen sich auf internationale Abkommen wie die UN-Resolution 1325 zu Frauen, Frieden und Sicherheit und deren Folgresolutionen. Doch gerade hier werden deren konzeptionelle Strukturprobleme deutlich. Fraglich ist, ob die Kombination des Kampfes gegen sexualisierte Gewalt mit den Forderungen nach politischer Partizipation von Frauen das geeignete Mittel ist, um Nachkriegsgesellschaften gerechter und gewaltfreier zu gestalten. Denn die Repräsentanz einzelner Frauen in Friedensverhandlungen oder Institutionen, etwa in Parlamenten, oder die Einrichtung eines Gender-Ministeriums sind keine hinreichenden Kriterien für Änderungen der politischen Machtstrukturen. Viele Politikerinnen sind marginalisiert, dennoch sehen sie sich nicht als Allianzpartnerinnen von Frauenorganisationen, sondern folgen eher der von männlichen Kollegen vorgegebenen Parteidisziplin. Dies ist vor allem dann der Fall, wenn Frauenorganisationen wie in Liberia zerstritten oder wie in der D.R. Kongo zu schwach und unzureichend vernetzt sind, um eine starke gemeinsame Lobby zu bilden. Möglicherweise kann der innerafrikanische Austausch, der gelegentlich von der europäischen Entwicklungszusammenarbeit gefördert wird, zu Problemlösungen und Perspektiverweiterungen der Friedensaktivistinnen beitragen. Ob allerdings die Ergebnisse solcher Dialoge die AU- oder UNEbenen erreichen, ist fraglich.

Die Herausforderung für diese zivilgesellschaftlichen Gruppen, für staatliche Institutionen und politische Entscheidungsträgerinnen besteht weiter darin, ihre Gender-Konzepte unter Berücksichtigung der multiplen Differenzen zwischen Frauen konstruktiv zu erweitern und neue Strategien zum Umgang mit Traditionsvertreterinnen bzw. -vertretern zu entwickeln. Zudem sind sie gefordert, kriegsgeprägte Maskulinität aufzuarbeiten. Daran müssten Männer in unterschiedlichen Führungsfunktionen mitwirken und Verantwortung als „Change Agents“ übernehmen. Ihre Aufgabe wäre es, im Austausch mit den Aktivistinnen Bezüge zu Geschlechtergerechtigkeit als sozialer Gerechtigkeit, zu Landrechten, Menschenrechten und der Verbesserung von Rechtssicherheit herzustellen - Ziele, die auch Männer direkt betreffen. Solche Transformationsansätze könnten im Idealfall zur Überwindung der in den UN-Resolutionen zu Frauen, Frieden und Sicherheit enthaltenen Fallstricke beitragen.

\section{Literatur}

Amnesty International 2004, Mass rape - Democratic Republic of Congo. London.

Baaz, Eriksson Maria/Stern, Maria 2009, Why do soldiers rape? Masculinity, violence, and sexuality in the armed forces in the D.R. Congo. in: International Studies Quarterly 53, 495-518.

Barnes, Karen 2011, The evolution and implementation of the United Nations Security Council Resolution 1325 on Women, Peace and Security. An overview. in: Olosakin, Funmi/Barnes, Karen/Ikpe, Eka (eds.): Women, peace and security. Translating policy into practice. London, 15-33.

Carpenter, Charli 2006, Innocent women and children. Gender norms, and the protection of civilians. Aldershot.

Chandler, Robin/Wang, Lihua/Fuller, Linda 2010 (eds.), Women, war and violence. Personal perspectives and global activism. New York.

Cleaver, Tessa/Wallace, Marion 1990, Namibia. Women and war. London.

Dolan, Chris 2010, War is not yet over. Community perceptions of sexual violence and its underpinnings in the Eastern DRC. London.

Eifler, Christina/Seifert, Ruth (eds.) 2009, Gender dynamics in post-conflict reconstruction. Frankfurt a.M.

Fuest, Veronika 2008, "This is the time to get in front". Changing roles and opportunities for women in Liberia. in: African Affairs 107/427, 201-224.

Human Rights Watch 2010, Always on the run. The vicious cycle of displacement in the Eastern Congo. New York.

Jacobs, Susie/Jacobson, Ruth/Marchbank, Jen (eds.) 2000, States of conflict. Gender, violence and resistance. London.

Karamé, Kari (ed.) 2004, Gender and peacebuilding in Africa. Oslo.

Kaufman, Joyce/Williams, Kristen 2010, Women and war. Gender identity and activism in times of conflict. Bloomfield.

Moser, Caroline/Clark, Fiona (eds.) 2001, Victims, perpetrators or actors? Gender, armed conflict and political violence. London. 
Myrttinen, Henri 2003, Disarming masculinities. in: Disarmament Forum 4, 37-46.

Nagelhus Schia, Niels/de Carvalho, Benjamin 2009, "Nobody gets justice here!" Addressing sexual and gender-based violence and the rule of law in Liberia, in: Conflict Trends 3, 26-33.

Olonisakin, Funmi/Okech, Awino 2011, Women and security governance in Africa. Cape Town.

Olsson, Louise/Tryggestad, Toruun (eds.) 2001, Women and international peacekeeping. London.

Schäfer, Rita 2008, Frauen und Kriege in Afrika. Ein Beitrag zur Gender-Analyse. Frankfurt a.M.

Puechguirbal, Nadine 2010, Discourses on gender, patriarchy and Resolution 1325. A textual analysis of UN documents. in: International Peacekeeping 17 (2) 172-187.

Shepherd, Laura 2008, Gender, violence and security. Discourse as practice. London.

Sjoberg, Laura/Via, Sandra (eds.) 2010, Gender, war and militarism. Feminist perspectives. Santa Barbara.

Tripp, Aila Mari/Casimiro, Isabel/Kwesiga, Joy Mungwa, Alice 2009, African women's movements. Changing political landscapes. Cambridge.

Wamai, Emma Njoki 2011, UNSCR 1325 implementation in Liberia. Dilemmas and challenges. in: Olosakin, Funmi/Barnes, Karen/Ikpe, Eka (eds.), Women, peace and security, Translating policy into practice. London, 52-65.

Wibben, Annick 2011, Feminist security studies. A narrative approach. London

Wisotzki, Simone 2011, Peace matters. Leerstellen in der Friedens- und Konfliktforschung. in: Femina Politica 1, 19-29.

\section{Kriegsforschung}

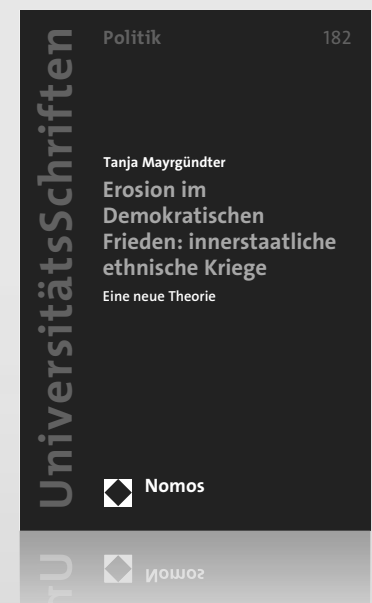

\section{Erosion im Demokratischen Frieden: innerstaatliche ethnische Kriege}

Eine neue Theorie

Von Dr. Tanja Mayrgündter

2012, 233 S., brosch., 39,- $€$

ISBN 978-3-8329-7134-2

(Nomos Universitätsschriften - Politik, $B d .182$ )

Der Demokratische Frieden ist ein vielfach diskutiertes Theorem, wobei das Phänomen der innerstaatlichen ethnischen Kriege in ausgereiften Demokratien bisher nicht berücksichtigt wurde. Dieser neue Aspekt wird in den Konfliktstrukturellen Ansatz und Erwartungen aufgenommen, um dann zu einer angemessenen Theorie des Demokratischen Friedens zu gelangen.

Weitere Informationen: www.nomos-shop.de/14278

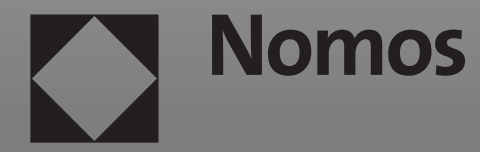

